\title{
Using Spatial Distribution Maps to Estimate APT Efficiency
}

\author{
B. P. Geiser, D. J. Larson, T. J. Prosa, T. F. Kelly, and R. M. Ulfig
}

CAMECA Instruments, Inc., 5500 Nobel Drive, Madison, WI 53711 USA

We describe a method for estimating system detection efficiency of atom probe tomography (APT) instruments that incorporates all losses in the system. Although, generally speaking, the spatial resolution in APT is on the order of a few angstroms, it is possible to observe three-dimensional, compositional information with resolution of the crystal lattice [1] and atomic efficiency greater than 50 per cent. In pure tungsten, for example, the three-dimensional crystallographic structure can be observed through large fractions of the analysis volume, Fig. 1a, and this can be used to estimate atomic detection efficiency.

The proposed method is based on analyzing reconstructed crystal volumes with a spatial distribution map (SDM) [2]. The SDM is a 3D map of the average neighbor positions around each detected atom and provides the ability to average over all atoms in the data set (shown in two dimensions below). Signal enhancement provided by the noise reduction of averaging results in the ability to clearly identify lattice locations in three dimensions.

We automatically perform a series of reconstruction adjustments, Figs. 1b, 2a, and 2b, to a tungsten analysis designed to produce an SDM by applying post-projection scale factors in the $\mathrm{X}, \mathrm{Y}$ and $\mathrm{Z}$ dimensions, and a shear correction (if necessary). We choose reconstruction parameters that maximize the signal-to-noise ratio of the Z SDM (peak-to-valley in Fig. 2c). Once the reconstruction is calibrated, all atoms within a single spacing in $\mathrm{X}, \mathrm{Y}$ and $\mathrm{Z}$, Fig. 2d, are counted around each atom treated as a center in the SDM. Then, efficiency can be calculated as:

$$
\varepsilon=\frac{N_{\text {Counts }}}{N_{\text {Centers }} \times N_{\text {Lattice_Spots }}} .
$$

System efficiencies of $59 \%$ have been measured.

References:

[1] B. Gault, M.P. Moody, F. De Geuser, A. La Fontaine, L.T. Stephenson, D. Haley, S.P. Ringer, Micro. Microanal. 16 (2010) 99.

[2] B.P. Geiser, T.F. Kelly, D.J. Larson, J. Schneir, J.P. Roberts, Micro. Microanal. 13 (2007) 437. 


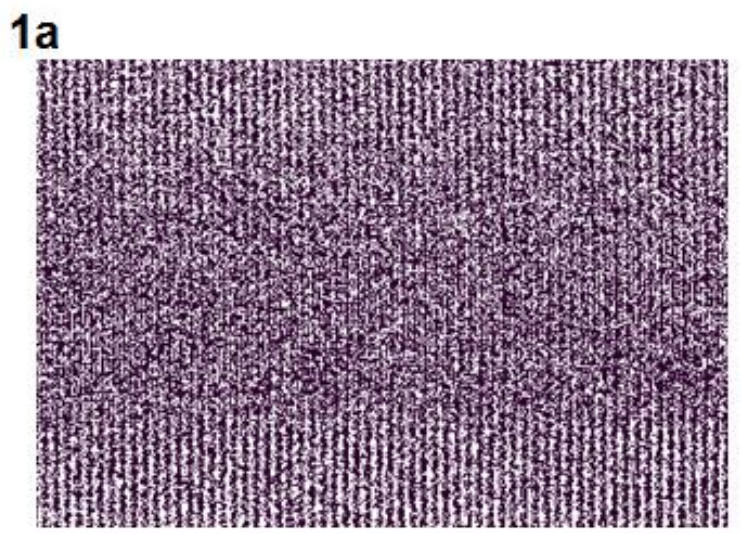

$1 \mathrm{~b}$
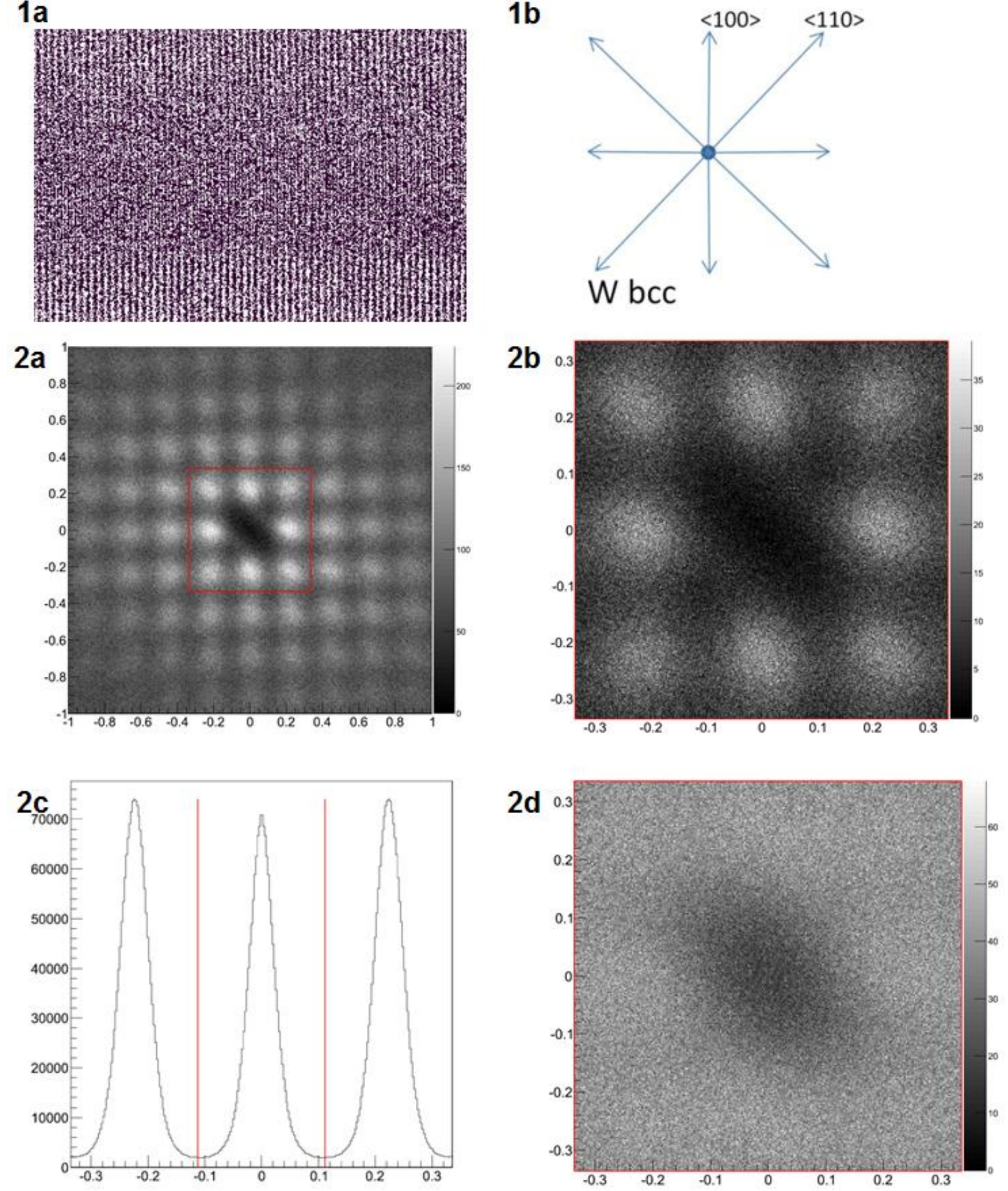

Fig. 1. (a) $<110>$ oriented tungsten is analyzed and reconstructed based on the parameters that maximize the Z SDM SNR. (b) The reconstruction is rescaled and reoriented so that the centermost 9 atoms (including the removed center atom) of the lattice fit into a square histogram region.

Fig. 2. (a) Shows the context of the SDM region to counted, (b) shows the extracted XY ROI, (c) shows the Z SDM and the cuts to be imposed, (d) shows the XY SDM that results from the relaxed Z cut. All spatial dimensions are in units of $\mathrm{nm}$. 